\title{
Welfare Equivalent NNP and Habit Formation*
}

\author{
Thomas Aronsson and Karl-Gustaf Löfgren \\ Department of Economics, Umeå University, \\ SE - 90187 Umeå, Sweden
}

April 2006

\begin{abstract}
This note concerns the importance of habit formation for social accounting. With internal habit formation, earlier procedures for welfare measurement in the first best apply with minor modifications. This result strengthens the idea behind using the comprehensive net national product as a welfare indicator. If, on the other hand, part of the habit formation is exogenous to each consumer, the exact welfare measure will also reflect the associated external effect.
\end{abstract}

\section{Introduction}

During the last 30 years, a theory a social accounting has gradually evolved. One of the main issues has been to establish a framework for measuring welfare at the national level and, in particular, to address the relationship between a measure of national welfare and the net national product (NNP). This has lead to the notion of comprehensive NNP, which is an extension of

*The authors would like to thank Tomas Sjögren for helpful comments and suggestions. A research grant from FORMAS is also gratefully acknowledged. 
the traditional NNP measure that reflects all relevant aspect of consumption and capital formation for society. In a first best setting with a stationary technology, Weitzman (1976) showed that the Hamiltonian of the underlying optimal growth problem - which is interpretable as the comprehensive NNP in utility terms - constitutes an exact welfare indicator in the sense of being proportional to the present value of future utility facing the representative consumer. More recent research has taken a variety of different directions; literature reviews are given by Weitzman (2003) and Aronsson et al. (2004).

A possible criticism of almost all literature on social accounting is that it is based on time-separable preferences. This stands in contrast to research in some other areas of economics, where the potential role of habit formation has been thoroughly addressed ${ }^{1}$. Earlier literature, which to a large extent focuses on consumption (and leisure) habits, admits a broad definition of habit formation; it may imply that the current instantaneous utility depends on one's own past consumption (internal habit formation) or on the past consumption of others (external habit formation). The purpose of this note is to introduce habit formation into an otherwise standard framework for social accounting and analyze the consequences of this extension for welfare measurement. We show that, if internal habit formation takes place in the context of a socially optimal resource allocation, a slightly modified version of Weitzman's (1976) welfare measure is applicable, which considerably strengthens the notion of the welfare equivalent, or comprehensive, NNP. If, on the other hand, part of the habit structure is exogenous to the individual, due to that habits are partly formed by earlier generations or the desire of catching-up with the Jonses (external habit formation), then the comprehensive NNP in utility terms fails as a welfare indicator; a result in accordance with earlier research on welfare measurement under market

\footnotetext{
${ }^{1}$ See e.g. Alessie and Lusardi (1997) and Alonso-Carrera et al. (2005) dealing with the theories of savings behavior and growth, respectively. See also the empirical studies on consumption by Dynan (2000) and Carrasco et al. (2005) as well as the references therein, and labor supply by Woittiez and Kapteyn (1998).
} 
failures.

We present the model and the main results in the next section. Section 3 concludes the paper.

\section{The Model and the Main Results}

Following much of the earlier literature on social accounting, we consider a representative agent economy with a fixed population and normalize the number of consumers to one. The instantaneous utility function takes the form

$$
u(t)=u(c(t), h(t))
$$

where $c$ is consumption and $h$ the stock of habits. The function $u(\cdot)$ is increasing in $c$, decreasing in $h$ and strictly concave. To begin with, we concentrate on internal habit formation, and assume that the stock of habits accumulates via present and past consumption, i.e.

$$
\dot{h}(t)=g(c(t), c(t-\tau))-\gamma h(t)
$$

where $h(0)=h_{0}$ and $\lim _{t \rightarrow \infty} h(t) \geq 0$, meaning the optimal control problem is characterized by a delayed response mechanism. The net investments at time $t$ are determined by

$$
\dot{k}(t)=f(k(t))-c(t)
$$

with $k(0)=k_{0}$ and $\lim _{t \rightarrow \infty} k(t) \geq 0$. The function $f(\cdot)$ measures the net output produced by labor (which is assumed to be constant and is, therefore, suppressed) and capital. The production function is strictly concave.

To derive the social optimum, it is convenient to assume that the resource allocation is decided upon by a social planner. The social planner's problem is to choose $c(t)$ such as to maximize 


$$
\int_{0}^{\infty} u(c(t), h(t)) e^{-\theta t} d t
$$

subject to equations (2) and (3) as well as subject to the initial and terminal condition. The present value Hamiltonian at time $t$ is written

$$
H(t)=u(c(t), h(t)) e^{-\theta t}+\lambda(t) \dot{k}(t)+\mu(t) \dot{h}(t)
$$

where $\lambda$ and $\mu$ are costate variables. In this particular framework, the first order condition for the control variable reads $\partial H(t) / \partial c(t)+\partial H(t+$ $\tau) / \partial c(t)=0$, whereas the equations of motion for the costate variables take the standard form ${ }^{2}$, i.e. $\dot{\lambda}(t)=-\partial H(t) / \partial k(t)$ and $\dot{\mu}(t)=-\partial H(t) / \partial h(t)$. Let $\left\{c^{*}(t)\right\}_{0}^{\infty}$ be the socially optimal path for the control variable, while $\left\{k^{*}(t), h^{*}(t)\right\}_{0}^{\infty}$ are the associated paths for the state variables.

By differentiating the present value Hamiltonian with respect to time, and then using the first order conditions described above together with $\lim _{t \rightarrow \infty} H^{*}(t)=0$, we are able to derive the welfare measure

$$
\theta \int_{t}^{\infty} u\left(c^{*}(s), h^{*}(s)\right) e^{-\theta(s-t)} d s=H^{c^{*}}(t)
$$

where $H^{c^{*}}(t)=H^{*}(t) e^{\theta t}$ is the current value Hamiltonian evaluated at the social optimum. We have derived the following result;

Proposition 1 With a fixed technology, and if the habits evolve internally in a socially optimal resource allocation, then welfare (the present value of future utility) is appropriately measured by the current value Hamiltonian implicit in the social planner's optimization problem.

To facilitate the interpretation of the current value Hamiltonian in terms of the comprehensive NNP, let us approximate $u(c, h)$ by a linear function as well as define the 'consumer surplus', $C S=u(c, h)-u_{c} c-u_{h} h$, where the

\footnotetext{
${ }^{2} \mathrm{~A}$ more thorough analysis of delayed response problems in optimal control theory is given by Kamien and Schwartz (1991).
} 
subindices denote partial derivatives. By using the first order condition for consumption together with the short notation $g(t)=g(c(t), c(t-\tau))$, the effective shadow price of $c(t)$ in current value terms can be written as

$$
\hat{\lambda}^{c}(t)=\lambda^{c}(t)-\mu^{c}(t) \frac{\partial g(t)}{\partial c(t)}-\mu^{c}(t+\tau) \frac{\partial g(t+\tau)}{\partial c(t)}=u_{c}(c(t), h(t))
$$

where $\lambda^{c}(t)=\lambda(t) e^{\theta t}$ and $\mu^{c}(t)=\mu(t) e^{\theta t}$. We can rewrite equation (6) as a welfare measure in real terms

$$
\begin{aligned}
\frac{\theta \int_{t}^{\infty} u\left(c^{*}(s), h^{*}(s)\right) e^{-\theta(s-t)} d s}{\hat{\lambda}^{c^{*}}(t)}= & c^{*}(t)+\rho^{*}(t) h^{*}(t)+q_{k}^{*}(t) \dot{k}^{*}(t) \\
& +q_{h}^{*}(t) \dot{h}^{*}(t)+\frac{C S^{*}(t)}{\hat{\lambda}^{c^{*}}(t)}
\end{aligned}
$$

in which $\rho=u_{h}(\cdot) / \hat{\lambda}^{c}, q_{k}=\lambda^{c} / \hat{\lambda}^{c}$ and $q_{h}=\mu^{c} / \hat{\lambda}^{c}$. The right hand side of equation (7) measures the sum of the real comprehensive NNP and the real consumer surplus. Therefore, with the modification that the effective shadow price of consumption at time $t$ reflects how $c(t)$ affects $H(t)$ and $H(t+\tau)$, equation (7) resembles results derived in the absence of the delayed response mechanism.

If, on the other hand, the consumer treats part of the stock of habits as exogenous, Proposition 1 does not apply ${ }^{3}$. For instance, the stock of habits may, in part, reflect the behavior of others (exemplifying the desire of catching-up with the Jonses). Without adding unnecessary complications to our model, let us take this argument to its extreme point by considering a resource allocation, $\left\{c^{0}(t)\right\}_{0}^{\infty}$, which satisfies $\partial H(t) / \partial c(t)=0$ at each instant. In other words, the consumer treats the consumption lag as if it is exogenous. Given this modification, the welfare measure changes to read

\footnotetext{
${ }^{3}$ This would also follow in the context of a first best resource allocation problem, in the more general case where the time-delay itself, i.e. $\tau$, is a time dependent function (see Kamien and Schwartz (1991) for such a decision problem). The reason is that a time dependent delay introduces another source of welfare change as time passes.
} 


$$
\theta \int_{t}^{\infty} u\left(c^{0}(s), h^{0}(s)\right) e^{-\theta(s-t)} d s=H^{c^{0}}(t)+\int_{t}^{\infty} \mu^{0}(s) \frac{\partial g^{0}(s)}{\partial c(s-\tau)} \dot{c}^{0}(s-\tau) e^{\theta t} d s
$$

where the superindex " 0 " is used to distinguish this resource allocation from the socially optimal resource allocation described above. We have derived;

Proposition 2If the habit formation is (at least in part) external, then welfare is measured by the sum of the current value Hamiltonian and the present value of changes in past external consumption measured over the future planning period.

Equation (8) relates, in a natural way, to earlier results on welfare measurement under external effects ${ }^{4}$, in the sense that the exact welfare measure contains forward looking terms. Here, if part of the current consumption is chosen without proper recognition of its future welfare consequences, then the shadow price of consumption implicit in the resource allocation (on which the current value Hamiltonian is based) differs from the corresponding 'social' shadow price. Therefore, we must add this information, which explains the forward looking term at the right hand side of equation (8).

\section{Summary and Conclusions}

This paper reconciles the growth theoretical literature on social accounting with the concept of habit formation. The results suggest that internal habit formation does not invalidate the welfare interpretation of the current value Hamiltonian. However, if the habits are, in part, determined by variables that are exogenous to each consumer, then welfare measurement necessitates more information than what is part of the current value Hamiltonian; in this case, the welfare measure also contains the present value of changes in the consumption lags (the welfare effects of which are not fully internalized with external habit formation).

\footnotetext{
${ }^{4}$ See e.g. Aronsson and Löfgren (1999).
} 


\section{References}

[1]Alessie, R. and Lusardi, A. (1997) Consumption, Saving and Habit Formation. Economics Letters 55, 103-108.

[2]Alonso-Carrera, J., Caballé, J. Raurich, X. (2005) Growth, Habit Formation and Catching-up with the Jonses. European Economic Review 49, 1665-1691.

[3]Aronsson, T. and Löfgren, K-G. (1999b) Pollution Tax Design and Green National Accounting. European Economic Review 43, 1457-1474.

[4]Aronsson, T., Löfgren, K-G. and Backlund, K. (2004) Welfare Measurement in Imperfect Markets: A Growth Theoretical Approach. Edward Elgar Publishing Limited.

[5]Carrasco, R., Labeaga, J.M., and López, D. (2004) Consumption and Habits: Evidence from Panel Data. Economic Journal 115, 144-165.

[6]Dynan, K. (2000) Habit Formation in Consumer Preferences: Evidence from Panel Data. American Economic Review 90, 391-406.

[7]Kamien, M. and Schwartz, N. (1991) Dynamic Optimization - The Calculus of Variations and Optimal Control in Economics and Management. Amsterdam: North-Holland.

[8]Weitzman, M.L. (1976) On the Welfare Significance of National product in a Dynamic Economy. The Quarterly Journal of Economics 90, 156162.

[9]Weitzman, M.L. (2003) Income, Capital, and the Maximum Principle. Harvard University Press: Cambridge, Massachusetts.

[10]Woittiez, I. and Kapteyn, A. (1998) Social Interaction and Habit Formation in a Model of Female Labor Supply. Journal of Public Economics 70, 185-205. 